\title{
Abstract
}

SLAC-PUB-6658

hep-ph/9409214

September, 1994

\section{Recent Progress in One-Loop Multi-Parton}

\section{Calculations *}

\section{Zvi Bern}

Department of Physics

University of California, Los Angeles

Los Angeles, CA 90024

Lance Dixon

Stanford Linear Accelerator Center

Stanford University, Stanford, CA 94309

David C. Dunbar

University College of Swansea

Swansea, Wales, United Kingdom

David A. Kosower

Service de Physique Théorique de Saclay.

Centre d'Etudes de Saclay

F-91191 Gif-sur-Yvette cedex, France

Presented by L.D. at the High-Energy Physics QCD Workshop 94, Montpellier, France,

$$
\text { July 7-13, } 1994
$$

* Research supported by the Department of Energy under grants DE-AC03-76SF00515 and DE-FG03 91ER40662, by the Alfred P. Sloan Foundation under grant BR-3222, by the National Science Foundation under grant PhY-9289, by the Commissariat a
We describe techniques that simplify the calculation of one-loop QCD amplitudes with many external legs, which are needed for next-to-leading-order (NLO) corrections to multi-jet processes. The constraints imposed by perturbative unitarity, collinear singularities and a supersymmetry-inspired organization of helicity amplitudes are particularly useful. Certain sequences of one-loop helicity amplitudes may be obtained for an arbitrary number of external gluons using these techniques. We also report on progress in completing the set of one-loop helicity amplitudes required for NLO threejet production at hadron colliders, namely the amplitudes with two external quarks and three gluons.

\section{DISCLAIMER}

This report was prepared as an account of work sponsored by an agency of the United States Government. Neither the United States Government nor any agency thereof, nor any of their employees, makes any warranty, express or implied, or assumes any legal liability or responsibility for the accuracy, completeness, or usefulness of any information, apparatus, product, or process disclosed, or represents that its use would not infringe privately owned rights. Reference herein to any specific commercial product, process, or service by trade name, trademark manufacturer, or otherwise does not necessarily constitute or imply its endorsement, recommendation, or favoring by the United States Government or any agency thereof. The views and opinions of authors expressed herein do not necessarily state or reflect those of the United States Government or any agency thereof.

\section{MAeten \\ DISTHA UTON OF NHS DOCUMANT W UWLATED}




\section{INTRODUCTION}

High- $p_{T}$ events in hadronic collisions and high-energy $e^{+} e^{-}$annihilations often produce a large number of jets. Quantitative QCD predictions for such multi-jet rates are important, but require at least next-to-leading-order (NLO) calculations. NLO corrections in turn require one-loop amplitudes with many external partons. The analytic complexity of oneloop calculations grows very rapidly with the number of external legs. As a result, complete NLO results are currently available only for processes with up to four external partons or vector bosons, such as $e^{+} e^{-} \rightarrow 3$ jets, and $p \tilde{p}$ (or $p p$ ) production of inclusive jets, di-jets, and $(W, Z)+1$ jet. To go further requires one-loop QCD amplitudes with five or more external partons or vector bosons. Here we briefly describe some very useful tools for carrying out such calculations, and summarize recent progress.

\section{ORGANIZATIONAL TOOLS}

The basic principle in calculating a complicated one-loop amplitude is to break it up into as many simpler, yet physical, pieces as possible. Traditional Feynman diagrams are not a good way to do this since individual diagrams are gauge-variant, and hence unphysical. Instead one should use the quantum numbers of the external particles, namely their helicities $( \pm)$ and color quantum-numbers, to decompose the amplitude into color-ordered helicity subamplitudes, or sub-amplitudes for short. The helicity [1] and color [2] decompositions of multi-parton tree-level amplitudes have been essential to their efficient calculation, and the same is true at one loop. The analytic properties of the one-loop sub-amplitudes - namely their collinear behavior (poles) and their unitarity properties (cuts) - are simpler than those of the full amplitude, yet they provide powerful constraints. They are simpler mainly because they only involve "color-adjacent" kinematic invariants such as $\left(k_{i}+k_{i+1}\right)^{2}$, where $k_{i}$ and $k_{i+1}$ are adjacent momenta with respect to the color ordering. Consequently the subamplitudes themselves tend to be simple; analytic expressions for each one-loop five-parton sub-amplitude takes up less than a page $[3,4]$, whereas the color-summed cross-section would fill hundreds of pages. Thus one should calculate sub-amplitudès analytically, and carry out the squaring of amplitudes and the sum over colors and helicities numerically at the very end.

The analytic properties of the sub-amplitudes can be exploited to simplify their calculation. Consider the constraints from perturbative unitarity. It is well known that the cuts (absorptive parts) of a loop amplitude are much easier to calculate than the full amplitude, because they are given by phase-space integrals of products of tree amplitudes [5]. The phase-space integrals can be performed to obtain integral functions with the correct cuts, omitting the need to do a dispersion integral [6]. Thus the full amplitude is easily reconstructed from the various cuts, up to additive "polynomial" terms (lacking branch cuts). It may be possible to determine the polynomial terms recursively using their collinear singularities (there is still a uniqueness question here). If a certain power-counting criterion holds - the degree of the loop-momentum polynomial for each diagram should be two fewer than the maximum possible in gauge theory - then there are actually no polynomial ambiguities and the sub-amplitudes can be completely reconstructed from the cuts [6,7]. Supersymmetric amplitudes provide examples satisfying the criterion; infinite sequences of "maximally helicity violating" $n$-gluon supersymmetric amplitudes can be efficiently calculated via their cuts $[6,7]$.

Direct calculation of sub-amplitudes can also be simplified using a decomposition based on the internal (spin) quantum numbers of particles going around the loop. For example, in a one-loop $n$-gluon amplitude the contribution of a gluon propagating around the loop would traditionally lead to a large amount of algebra, due to the complicated non- 


\section{DISCLAIMER}

Portions of this document may be illegible in electronic image products. Images are produced from the best available original document. 
abelian self-interaction vertex. However, it is possible to rewrite the gluon self-interaction (using either background-field gauge [8] or a string-based approach [9]) so that the gluon in the loop looks like a scalar in the loop, plus "a little bit more". One can rewrite [3],

$$
\begin{aligned}
\text { gluon }= & \text { scalar } \\
& -4 \times[\text { fermion }+ \text { scalar }] \\
& +[\text { gluon }+4 \times \text { fermion }+3 \times \text { scalar }]
\end{aligned}
$$

where all entries correspond to two-component fields circulating in the loop (gluons, Weyl fermions, complex scalars). The "little bit more" represented by the last two lines is supersymmetric (the contribution of $4 N=1$ chiral multiplets, and of an $N=4$ super-Yang-Mills theory), and is calculable via its cuts. In this way the gluon computation can be traded for the easier scalar case.

Recursive techniques, which were first applied at tree-level by Berends and Giele [10, 11], are now beginning to show promise at loop-level. Mahlon [12] has recently obtained two infinite sequences of one-loop $n$-gluon amplitudes by "sewing up" recursively determined off-shell tree amplitudes.

\section{EXPLICIT RESULTS}

What are the practical consequences of these tools so far? At the one-loop five-parton level, they have been used to calculate the full set of helicity amplitudes for five external gluons [3] and for two quarks and three gluons (which are almost complete [13]). In contrast, the calculation of the four-quark one-gluon amplitudes used (of the above tools) only the sub-amplitude decomposition [4].

Here we present one of the $\bar{q} q g g g$ partial amplitudes, $A_{5 ; 1}\left(1_{\bar{q}}^{-}, 2_{q}^{+}, 3^{-}, 4^{+}, 5^{+}\right)$, which is the coefficient of the color structure $N_{c}\left(T^{a_{3}} T^{a_{4}} T^{a_{5}}\right)_{i_{2}}^{i_{1}}$ for the indicated helicity assignments; particles 3,4,5 are gluons. For the gauge group $S U\left(N_{c}\right)$ with $n_{f}$ flavors of quark and $n_{s}$ flavors of scalar quarks, $A_{5 ; 1}$ can be decomposed into primitive amplitudes, from which all factors of $N_{c}, n_{f}, n_{s}$ have been extracted, as:

$$
\begin{aligned}
& A_{5 ; 1}\left(1_{\bar{q}}^{-}, 2_{q}^{+} ; 3^{-}, 4^{+}, 5^{+}\right) \\
& =\left(1+\frac{1}{N_{c}^{2}}\right) A_{5}^{L}\left(1_{\bar{q}}^{-}, 2_{q}^{+}, 3^{-}, 4^{+}, 5^{+}\right)-\frac{1}{N_{c}^{2}} \frac{\langle 32\rangle}{\langle 31\rangle} A_{5 ; 1}^{\mathrm{SUSY}}\left(1^{-}, 2^{+}, 3^{-}, 4^{+}, 5^{+}\right) \\
& -\left(\frac{n_{f}}{N_{c}}+\frac{1}{N_{c}^{2}}\right) A_{5}^{f}\left(1_{\bar{q}}^{-}, 2_{q}^{+} ; 3^{-}, 4^{+}, 5^{+}\right)+\left(\frac{n_{s}-n_{f}}{N_{c}}-\frac{1}{N_{c}^{2}}\right) A_{5}^{s}\left(1_{\bar{q}}^{-}, 2_{q}^{+} ; 3^{-}, 4^{+}, 5^{+}\right)
\end{aligned}
$$

where $A_{5 ; 1}^{\text {SUSY }}\left(1^{-}, 2^{+}, 3^{-}, 4^{+}, 5^{+}\right)$is the pure super-Yang-Mills amplitude for five external gluons, taken from ref. [3]. In the remaining components, the poles in $\epsilon$ in $D=4-2 \epsilon$ dimensional reduction are separated out into " $V$ " pieces by

$$
A_{5}^{x}=c_{\Gamma}\left(V^{x} A_{5}^{\text {tree }}+i F^{x}\right), \quad x=L, f, s
$$

where

$$
\begin{aligned}
c_{\Gamma} & =\frac{1}{(4 \pi)^{2-\epsilon}} \frac{\Gamma(1+\epsilon) \Gamma^{2}(1-\epsilon)}{\Gamma(1-2 \epsilon)}, \\
A_{5}^{\text {tree }} & =i \frac{\langle 13\rangle^{3}\langle 23\rangle}{\langle 12\rangle\langle 23\rangle\langle 34\rangle\langle 45\rangle\langle 51\rangle},
\end{aligned}
$$

and

$$
\begin{aligned}
V^{L}= & -\frac{1}{\epsilon^{2}} \sum_{j=2}^{5}\left(\frac{\mu^{2}}{-s_{j, j+1}}\right)^{\epsilon}+\sum_{j=1}^{5} \ln \left(\frac{-s_{j, j+1}}{-s_{j+1, j+2}}\right) \ln \left(\frac{-s_{j+2, j-2}}{-s_{j-2, j-1}}\right) \\
& +\frac{5}{6} \pi^{2}-\frac{3}{2 \epsilon}\left(\frac{\mu^{2}}{-s_{34}}\right)^{\epsilon}+\ln \left(\frac{-s_{51}}{-s_{12}}\right)-3, \\
V^{f}= & V^{s}=0 .
\end{aligned}
$$


The finite terms possess all the analytic complexity, and are given by

$$
\begin{aligned}
& F^{L}=F^{s}-\frac{\langle 12\rangle\langle 23\rangle\langle 34\rangle\langle 41\rangle[24]^{3} \operatorname{Ls}_{2}\left(\frac{-s_{23}}{-s_{51}}, \frac{-s_{34}}{-s_{51}}\right)}{s_{51}^{3}} \\
& -\frac{\langle 12\rangle\langle 23\rangle\langle 35\rangle^{2}[25]^{3}}{\left\langle\mathrm{Ls}_{2}\left(\frac{-s_{12}}{-s_{34}}, \frac{-s_{51}}{-s_{34}}\right)\right.} s_{34}^{3} \\
& -2 \frac{\langle 13\rangle\langle 23\rangle\langle 41\rangle[24]^{2}}{\langle 45\rangle\langle 51\rangle} \frac{L s_{1}\left(\frac{-s_{23}}{-s_{51}}, \frac{-s_{34}}{-s_{51}}\right)}{s_{51}^{2}}-2 \frac{\langle 13\rangle\langle 23\rangle\langle 35\rangle[25]^{2} L s_{1}\left(\frac{-s_{12}}{-s_{34}}, \frac{-s_{51}}{-s_{34}}\right)}{s_{34}^{2}} \\
& -\frac{\langle 13\rangle^{2}[24]}{\langle 45\rangle\langle 51\rangle} \frac{L s_{0}\left(\frac{-s_{23}}{-s_{51}}, \frac{-s_{34}}{-s_{51}}\right)}{s_{51}}-\frac{\langle 13\rangle^{2}\langle 35\rangle[25]}{\langle 34\rangle\langle 45\rangle\langle 51\rangle} \frac{\mathrm{Ls}_{0}\left(\frac{-s_{12}}{-s_{34}}, \frac{-s_{51}}{-s_{34}}\right)}{s_{34}} \\
& -\left(\frac{\langle 13\rangle\langle 23\rangle^{2}[25]^{2}\langle 15\rangle}{\langle 12\rangle\langle 34\rangle\langle 45\rangle}+\frac{1}{2} \frac{\langle 13\rangle^{2}[12]\langle 23\rangle[25]}{\langle 34\rangle\langle 45\rangle}\right) \frac{L_{1}\left(\frac{-s_{34}}{-s_{51}}\right)}{s_{51}^{2}} \\
& +\frac{1}{2} \frac{\langle 13\rangle\langle 14\rangle\langle 23\rangle[24]^{2} L_{1}\left(\frac{-s_{23}}{-s_{51}}\right)}{s_{51}^{2}}-\frac{1}{2} \frac{\langle 13\rangle\langle 15\rangle\langle 34\rangle[45]^{2}}{\langle 15\rangle\langle 51\rangle} \frac{L_{1}\left(\frac{-s_{12}}{-s_{34}}\right)}{s_{34}^{2}} \\
& +\frac{1}{2} \frac{\langle 13\rangle^{2}[24]}{\langle 45\rangle\langle 51\rangle} \frac{L_{0}\left(\frac{-s_{34}}{-s_{51}}\right)}{s_{51}}-\left[2 \frac{\langle 13\rangle^{2}[45]}{\langle 12\rangle\langle 45\rangle}+\frac{\langle 13\rangle^{2}\langle 35\rangle[25]}{\langle 34\rangle\langle 45\rangle\langle 51\rangle}\right] \frac{L_{0}\left(\frac{-s_{12}}{-s_{34}}\right)}{s_{34}} \\
& +\frac{1}{2} \frac{\langle 14\rangle[24]^{2}[45]}{\langle 45\rangle[23][34] s_{51}}-\frac{\langle 13\rangle\langle 23\rangle[25][45]}{\langle 12\rangle s_{34}\langle 45\rangle[51]}-\frac{1}{2} \frac{\langle 13\rangle^{2}[12]\langle 23\rangle[25]}{s_{34}\langle 34\rangle\langle 45\rangle s_{51}} \\
& F^{s}=\frac{1}{3}\left[\frac{\langle 15\rangle[25]\langle 34\rangle\langle 35\rangle[45]^{2}}{\langle 45\rangle} \frac{2 \mathrm{~L}_{2}\left(\frac{-s_{12}}{-s_{34}}\right)}{s_{34}^{3}}-\frac{\langle 13\rangle\langle 15\rangle\langle 34\rangle[45]^{2} \mathrm{~L}_{1}\left(\frac{-s_{12}}{-s_{34}}\right)}{s_{34}^{2}}\right. \\
& \left.-\frac{\langle 13\rangle[24][45]}{\langle 12\rangle[12][34]\langle 45\rangle}+\frac{[24]^{2}[25]}{[12][23][34]\langle 45\rangle}\right] \text {, } \\
& F^{f}=-\frac{\langle 13\rangle^{2}[45]}{\langle 12\rangle\langle 45\rangle} \frac{L_{0}\left(\frac{-s_{12}}{-s_{34}}\right)}{s_{34}}
\end{aligned}
$$

where the logarithms and dilogarithms $\left(\mathrm{Li}_{2}\right)$ are contained in

$$
\begin{aligned}
\mathrm{Lo}_{0}(r) & =\frac{\ln (r)}{1-r}, \quad \mathrm{~L}_{1}(r)=\frac{\mathrm{L}_{0}(r)+1}{1-r}, \quad \mathrm{~L}_{2}(r)=\frac{\ln (r)-\frac{1}{2}(r-1 / r)}{(1-r)^{3}} \\
\mathrm{Ls}_{0}\left(r_{1}, r_{2}\right) & =\frac{\mathrm{Li}_{2}\left(1-r_{1}\right)+\mathrm{Li}_{2}\left(1-r_{2}\right)+\ln r_{1} \ln r_{2}-\frac{\pi^{2}}{6}}{\left(1-r_{1}-r_{2}\right)} \\
\mathrm{Ls}_{1}\left(r_{1}, r_{2}\right) & =\frac{\mathrm{Ls}_{0}\left(r_{1}, r_{2}\right)+\mathrm{L}_{0}\left(r_{1}\right)+\mathrm{L}_{0}\left(r_{2}\right)}{\left(1-r_{1}-r_{2}\right)} \\
\mathrm{Ls}_{2}\left(r_{1}, r_{2}\right) & =\frac{\mathrm{Ls}_{1}\left(r_{1}, r_{2}\right)+\left(\mathrm{L}_{1}\left(r_{1}\right)+\mathrm{L}_{1}\left(r_{2}\right)\right) / 2}{\left(1-r_{1}-r_{2}\right)}
\end{aligned}
$$

Once the full set of $\bar{q} q g g g$ helicity amplitudes are available (roughly speaking, six expressions of the above type are required), numerical programs can be constructed for NLO three-jet production at hadron colliders. There are various general formalisms available [14, 15] for combining $(n+1)$-parton tree contributions and $n$-parton loop contributions into a NLO correction; the one of Giele, Glover, and Kosower is convenient because it is in a colorordered framework which meshes well with a color-ordered decomposition of the amplitudes.

\section{FUTURE PROSPECTS}

Projecting into the future, it seems that recently developed tools - especially the combination of unitarity, collinear singularities and recursive techniques - will make the calculation of one-loop six-parton and perhaps even seven-parton amplitudes quite practical within the next couple of years. Indeed, we expect that the bottleneck in getting NLO results out will shift from the analytical to the numerical end of the process. On the analytical side, the emphasis should shift (perhaps fairly soon) to two-loop multi-parton calculations, which are needed for next-to-next-to-leading (NNLO) results, such as the NNLO correction to 
$e^{+} e^{-}$annihilation to 3 jets, a result which could significantly reduce the theoretical error in determining $\alpha_{s}$ at the $Z$ pole.

\section{References}

[1] F.A. Berends, R. Kleiss, P. De Causmaecker, R. Gastmans and T.T. Wu, Phys. Lett. B103:124 (1981); P. De Causmaeker, R. Gastmans, W. Troost and T.T. Wu, Nucl. Phys. B206:53 (1982); R. Kleiss and W.J. Stirling, Nucl. Phys. B262:235 (1985); J.F. Gunion and Z. Kunszt, Phys. Lett. B161:333 (1985); R. Gastmans and T.T. Wu, The Ubiquitous Photon: Helicity Method for QED and QCD (Clarendon Press, 1990); Z. Xu, D.-H. Zhang and L. Chang, Nucl. Phys. B291:392 (1987).

[2] J.E. Paton and H.M. Chan, Nucl. Phys. B10:516 (1969); F.A. Berends and W.T. Giele, Nucl. Phys. B294:700 (1987); M. Mangano, Nucl. Phys. B309:461 (1988).

[3] Z. Bern, L. Dixon and D.A. Kosower, Phys. Rev. Lett. 70:2677 (1993).

[4] Z. Kunszt, A. Signer and Z. Trócsányi, preprint ETH-TH/94-14, hep-ph/9405386.

[5] L.D. Landau, Nucl. Phys. 13:181 (1959); S. Mandelstam, Phys. Rev. 112:1344 (1958), 115:1741 (1959); R.E. Cutkosky, J. Math. Phys. 1:429 (1960).

[6] Z. Bern, D.C. Dunbar, L. Dixon and D.A. Kosower, preprint SLAC-PUB-6415, hepph/9403226, to appear in Nucl. Phys. B.

[7] Z. Bern, D.C. Dunbar, L. Dixon and D.A. Kosower, preprint SLAC-PUB-6563.

[8] G. 't Hooft, in Functional and Probabilistic Methods in Quantum Field Theory, Vol. 1 (1975); B.S. DeWitt, in Quantum gravity II, eds. C. Isham, R. Penrose and D. Sciama
(Oxford, 1981); L.F. Abbott, Nucl. Phys. B185:189 (1981); L.F. Abbott, M.T. Grisaru and R.K. Schaefer, Nucl. Phys. B229:372 (1983).

[9] Z. Bern and D.A. Kosower, Phys. Rev. Lett. 66:1669 (1991); Nucl. Phys. B379:451 (1992); Z. Bern and D.C. Dunbar, Nucl. Phys. B379:562 (1992).

[10] F.A. Berends and W.T. Giele, Nucl. Phys. B306:759 (1988).

[11] D.A. Kosower, Nucl. Phys. B335:23 (1990).

[12] G.D. Mahlon, Phys. Rev. D49:4438 (1994).

[13] Z. Bern, L. Dixon and D.A. Kosower, in preparation.

[14] Z. Kunszt and D. Soper, Phys. Rev. D46:192 (1992); Z. Kunszt, A. Signer and Z. Trócsányi, Nucl. Phys. B420:550 (1994).

[15] W.T. Giele and E.W.N. Glover, Phys. Rev. D46:1980 (1992); W.T. Giele, E.W.N. Glover and D.A. Kosower, Nucl. Phys. B403:633 (1993). 